\title{
Assessment of Burnout among Canadian Pharmacists Working in Team-based Primary Care Settings
}

Hayley Bessette, BSc; Caitlin Chew, BSc; Anita I. Kapanen, MSc, PhD; Jillian Reardon, BSc(Pharm), PharmD, ACPR;

Jamie Yuen, BSc(Pharm), BCGP

Faculty of Pharmaceutical Sciences, The University of British Columbia, Vancouver, BC, Canada

\section{ABSTRACT}

Background: Burnout syndrome is well-documented among healthcare professionals across various practice settings. There has been recent expansion of Canadian pharmacists into team-based primary care and burnout in this setting has not been assessed. Our objective was to assess workplace burnout and to identify factors that play a role in perpetuating or diminishing it.

Method: An online survey to assess burnout was developed using the Maslach Burnout Inventory (MBI) tool and questions regarding pharmacist background and practice. Invitations to complete the survey were sent to Canadian pharmacists working in team-based primary care settings on November 26, 2019 via a national primary healthcare listserv.

Results: A total of 31/433 completed responses were collected. The main analysis focused on the personal accomplishment (PA) domain as it had an adequate response rate. The PA domain had a median score of 5.0 (95\% Cl 4.69-5.22). We compared medians of the PA domain across different groups of each categorical variable. We found that the number of years working in primary care settings was positively associated with a higher PA domain score $(p=0.029)$.

Discussion: PA was higher in pharmacists who have been practicing in a primary care setting for longer; however, burnout rates could not be properly assessed due to the limited response rate.

Conclusion: This is the first study to assess burnout among Canadian team-based primary care pharmacists. Personal accomplishment was higher in those who have been practicing in a primary care setting for longer. Future studies should consider alternate methods to evaluate burnout in this population.

Keywords: burnout, primary care, occupational stress

\section{INTRODUCTION}

Burnout syndrome, or occupational burnout is characterized as the manifestation of physical and emotional symptoms secondary to unresolved conflict, or job-related stress. ${ }^{1}$ Signs and symptoms of burnout can include increased frustration, anger, and even characteristics similar to those of clinical depression. ${ }^{2}$ More recently, with the increasing prevalence of job-related stress, the World Health Organization (WHO) officially classified burnout as an "occupational phenomenon". ${ }^{3}$ Burnout syndrome is broken down into three facets: 1) emotional exhaustion defined as the depletion of emotional energy secondary to workplace burnout, 2) depersonalization defined as the negativity and cynicism surrounding an individual's workplace or occupation, and 3) the decreased sense of personal accomplishment. ${ }^{4}$ Using these three facets, burnout can be characterised using the Maslach Burnout Inventory $(\mathrm{MBI})$, a validated tool created to measure and identify occupational burnout. ${ }^{4}$

Burnout syndrome among healthcare professionals is common as shown in existing literature. ${ }^{2}$ While current literature highlights burnout syndrome experienced by pharmacists working in academia, health-systems, hospital practice, and community practice, burnout rates among pharmacists practicing in primary care settings have not been explored. ${ }^{5-9}$

Corresponding author: Jamie Yuen, BSc(Pharm), BCGP

Faculty of Pharmaceutical Sciences

The University of British Columbia

2405 Wesbrook Mall, Vancouver, BC, Canada, V6T $1 Z 3$

Phone: 604-827-0704; Email: jamieh.yuen@ubc.ca
More pharmacists are transitioning into team-based primary care practice in Canada, resulting in changes to workplace settings, roles, and responsibilities. ${ }^{10,11}$ Our primary objective was to assess workplace burnout in Canadian primary care pharmacists and to identify factors that play a role in perpetuating or diminishing occupational burnout across the country.

\section{METHODS}

\section{Study Design}

Pharmacists practicing in a team-based primary care setting were sent a single invitation to participate in the study through a national primary healthcare listserv hosted by the Canadian Pharmacists Association (CPhA). An email invitation was sent to 433 registered users on November 26, 2019 and the survey closed on December 31, 2019. As any member of the CPhA can join the listserv, participants were required to self-identify as practicing in a team-based primary care setting prior to opening the survey. To determine rates of burnout, we created an anonymous, three-part online survey. Part one gathered general information about the pharmacist and their work. Part two used the $\mathrm{MBI}$ - Human Services Survey for Medical Personnel (MBI-HSS (MP)), which is specifically designed to assess burnout among healthcare professionals. ${ }^{12}$ This tool consists of 22 statements of job-related feelings which are assigned a score of 0 to 6 based on how often respondents experience each feeling. A scoring key is provided by MBI. ${ }^{13}$ Part three asked additional questions specific to work and non-work activities. 
The survey was created and hosted on the University of British Columbia Survey Tool (by Qualtrics.com). All surveys were completed using a distributed link. Participants provided informed consent by choosing to complete the survey. All survey data was anonymous.

\section{Data Analysis}

Data were interpreted according the MBI Manual. ${ }^{13}$ It has been documented by the authors of the MBI that cut-offs published in earlier editions of the MBI Manual had no diagnostic validity and were therefore removed. ${ }^{14}$ Because of this, stratifying of domain scores was not performed. Normal distribution of continuous data ( $\mathrm{MBI}$ domain scores) was assessed with the Shapiro-Wilk test. Kendall's tau and Spearman's rho tests were used for assessing correlation between the three domains. All categorical data, including participant age, are presented as percentages. Attributes of too small cell sizes (less than 5) were decoded and merged into reasonably sized subgroups for analysis. Non-parametric tests for pairwise comparison of medians of outcomes and attributes were used and p-values of $<0.05$ were considered statistically significant findings. SPSS version 26 (IBM) was used to assist statistical analysis.

\section{RESULTS}

At the closing of the online survey on December 31, 2019, 39 responses were collected ( $9 \%$ response rate). Out of these, four were incomplete, two stated their occupation as hospital pharmacists, two completed only the demographics questions but not the $\mathrm{MBI}$ tool, leaving 31 (7\%) responses eligible for analysis. Of those who responded, $84 \%$ are female with a median age range of $45-54$ years old and a median of 6-10 years practicing in primary care. Eighty-seven percent of respondents spend less than five hours a week on professional activities outside of the workplace and $77 \%$ spend greater than or equal to five hours on non-professional activities outside of the workplace. Forty one percent of respondents spend $75 \%$ or more of their work hours performing direct patient care and the majority of respondents (52\%) spend less than a quarter of their work hours completing documentation. Of the respondents, $45 \%$ reported burnout resources available to them at work and of those, only $8 \%$ have utilized these resources. Additional characteristics of the 31 respondents are listed in Table 1 and Table 2.

\begin{tabular}{|c|c|}
\hline Characteristic & No. of participants (\%) $\mathrm{N}=31$ \\
\hline \multicolumn{2}{|l|}{ Age } \\
\hline $25-34$ years & $8(25.8)$ \\
\hline $35-44$ years & $6(19.4)$ \\
\hline $45-54$ years & $10(32.3)$ \\
\hline $55-64$ years & $6(19.4)$ \\
\hline 65 years or older & $1(3.2)$ \\
\hline \multicolumn{2}{|l|}{ Gender } \\
\hline Male & $5(16.1)$ \\
\hline Female & 26 (83.9) \\
\hline Transgender & $0(0)$ \\
\hline Other & $0(0)$ \\
\hline Prefer not to say & $0(0)$ \\
\hline \multicolumn{2}{|l|}{ Level of pharmacy training ${ }^{\alpha}$} \\
\hline Bachelor of Science (Pharmacy) & $27(55.1)$ \\
\hline Entry-to-practice PharmD & $5(10.2)$ \\
\hline Graduate PharmD & $3(6.1)$ \\
\hline Accredited Pharmacy Residency & $12(24.5)$ \\
\hline Other & $2(4.1)$ \\
\hline \multicolumn{2}{|c|}{ Years of practice as a licensed pharmacist } \\
\hline Less than 1 & $2(6.5)$ \\
\hline $1-5$ & $3(9.7)$ \\
\hline $6-10$ & $4(12.9)$ \\
\hline $11-15$ & $3(9.7)$ \\
\hline Greater than 15 & $19(61.3)$ \\
\hline \multicolumn{2}{|c|}{ Year practicing in a primary care setting } \\
\hline Less than 1 & $2(6.5)$ \\
\hline $1-5$ & $7(22.6)$ \\
\hline $6-10$ & $14(45.2)$ \\
\hline $11-15$ & $5(16.1)$ \\
\hline Greater than 15 years & $3(9.7)$ \\
\hline
\end{tabular}




\begin{tabular}{lc}
\hline Current role ${ }^{\beta}$ & \\
Clinical Pharmacist & $26(83.9)$ \\
Academic Detailing ${ }^{\nu}$ & $1(3.2)$ \\
Program Management & $2(6.5)$ \\
\hline Current employment status & $21(67.7)$ \\
Full-time & $10(32.3)$ \\
Part-time & $2(6.5)$ \\
\hline Average hours a week worked & $2(6.5)$ \\
$0-9$ & $3(9.7)$ \\
$10-19$ & $16(51.6)$ \\
$20-29$ & $7(22.6)$ \\
$30-39$ & $1(3.2)$ \\
$40-49$ & $22(71.0)$ \\
Greater than 50 & $4(12.9)$ \\
\hline Relationship status & $5(16.1)$ \\
Married & \\
Single & $9(29.0)$ \\
Common-law & $3(9.7)$ \\
\hline Number of children & $17(54.8)$ \\
0 & $2(6.5)$ \\
1 & \\
2 & \\
Greater than 3 & \\
\hline${ }^{\alpha} 15$ respondents reported having more than one level of training \\
${ }^{3} 4$ respondents reported currently holding more than one role/position \\
${ }^{v}$ refers to role where pharmacists review current available evidence for select medication therapy topics with \\
physicians, nurses, and pharmacists
\end{tabular}

Table 2. Additional work and personal life characteristics.

\begin{tabular}{lc} 
Characteristic & No. of participants (\%) $\mathbf{N = 3 1}$ \\
\hline Hours a week spent on professional activities outside of the workplace & \\
Less than 5 & $27(87.1)$ \\
$5-10$ & $2(6.5)$ \\
Greater than 10 & $2(6.5)$ \\
\hline Hours a week spent on non-professional activities outside of the workplace & \\
Less than 5 & $7(22.6)$ \\
$5-10$ & $12(38.7)$ \\
Greater than 10 & $12(38.7)$ \\
\hline Current positions with involvement in the following activities: & \\
Pharmacy committee participation & $10(7.9)$ \\
Medical staff committee participation & $8(6.4)$ \\
Daily rounding & $2(1.6)$ \\
Administrative tasks & $22(17.5)$ \\
Residency precepting & $13(10.3)$ \\
Directing/coordinating a residency program & $1(0.8)$ \\
Research/publication & $7(5.6)$ \\
Medication reconciliation & $24(19.1)$ \\
Formal pharmacy consultation & $23(18.3)$ \\
Nutrition support consultation & $7(5.6)$ \\
Didactic lecturing & $9(7.1)$ \\
\hline
\end{tabular}




\begin{tabular}{|c|c|}
\hline \multicolumn{2}{|c|}{ Average percent of work hours spent performing direct patient care } \\
\hline Less than 25 & $5(16.1)$ \\
\hline $25-49$ & $5(16.1)$ \\
\hline $50-74$ & $8(25.8)$ \\
\hline 75 or more & $13(41.9)$ \\
\hline \multicolumn{2}{|c|}{ Average percent of work hours spent completing patient workups } \\
\hline Less than 25 & $15(48.4)$ \\
\hline $25-49$ & $8(25.8)$ \\
\hline $50-74$ & $5(16.1)$ \\
\hline 75or more & $3(9.7)$ \\
\hline \multicolumn{2}{|c|}{ Average percent of work hours spent completing documentation } \\
\hline Less than 25 & $16(51.6)$ \\
\hline $25-49$ & $12(38.7)$ \\
\hline $50-74$ & $2(6.5)$ \\
\hline 75 or more & $1(3.2)$ \\
\hline \multicolumn{2}{|c|}{ Average percent of work hours spent completing non-direct patient care tasks } \\
\hline Less than 25 & $19(61.3)$ \\
\hline $25-49$ & $7(22.6)$ \\
\hline $50-74$ & $2(6.5)$ \\
\hline 75 or more & $3(9.7)$ \\
\hline \multicolumn{2}{|c|}{ Burnout resources availability at work } \\
\hline Yes & $14(45.2)$ \\
\hline No & $6(19.4)$ \\
\hline Unsure & $11(35.5)$ \\
\hline \multicolumn{2}{|c|}{ Utilization of burnout resources if available ${ }^{\alpha}$} \\
\hline Yes & $1(7.7)$ \\
\hline No & $12(92.3)$ \\
\hline
\end{tabular}

The $\mathrm{MBI}$ tool completion rate was $90 \%$ for the personal accomplishment (PA) domain. Other domains (depersonalization and emotional exhaustion) were $70 \%$ and $45 \%$ complete, respectively. Analysis of nonparametric correlations showed the responses were consistent between the domains. Further analysis focused on the PA domain. The 31 responses analysed included two cases missing responses to two statements and one case missing a response to one statement. Due to our limited sample size, these three incomplete surveys were included in the analysis. Because of this, an averaged domain score was used instead of a sum of the domain scores.

The PA domain had a median score of 5.0 (95\% Cl 4.69-5.22). We compared medians of the PA domain across different groups of each categorical variable. Due to the small sample size, we chose to compare medians that are less influenced by extreme values than means would be. Results of the comparison analysis are summarized in Table 3. The number of years working in primary care settings was positively associated with a higher PA domain score $(p=0.029)$. No other attribute comparison in the PA domain had statistical significance.
Table 3. Comparison of medians of the Personal Achievement subdomain and character groups. Outcome: Personal Achievement (PA) Independent samples Median tests

\begin{tabular}{ll} 
Attribute $(\mathbf{n = 3 0})$ & p-value \\
\hline Age & .689 \\
Gender & .165 \\
Years in primary care & $.029^{\alpha}$ \\
Years in practice as licensed pharmacist & .302 \\
Average hours of week worked & .688 \\
Number of pharmacy degrees & .268 \\
Any PharmD & .877 \\
Accredited pharmacy residency & .412 \\
\hline aPairwise comparisons, 2-sided tests, asymptotic significances
\end{tabular}

\section{DISCUSSION}

With the increasing transition to team-based primary care in Canada, primary care pharmacists are practicing in novel work environments, taking on new roles, and facing unique challenges. Primary care pharmacists collaborate with patients and other care providers in the long-term management of medication-related problems and chronic diseases in an interprofessional setting. ${ }^{10,11} \mathrm{~A}$ recent commentary highlights burnout syndrome identified through a survey investigating physician professional satisfaction. The authors hypothesized that similar factors contributing to burnout in physicians may 
also apply to pharmacists. This may be due to similarities in work environment and the tightly regulated nature of these professions contributing to increase in workplace burnout. ${ }^{2} \mathrm{~A}$ systematic review evaluating burnout in physicians found a prevalence from $0 \%-86.2 \%$, underscoring the varied rates of burnout among physicians. ${ }^{15}$ The rates of burnout previously observed in pharmacists practicing in academia showed $41.3 \%$ of participants experiencing emotional exhaustion. ${ }^{5}$ Further, burnout studied among health-system pharmacists showed that $53.2 \%$ of participants experienced a high degree of burnout in one of three facets of occupational burnout. ${ }^{7}$ To our knowledge, there are no published studies assessing occupational burnout in Canadian community or hospital pharmacists. However, a Canadian study evaluating community and hospital pharmacists' perceptions of working conditions showed that pharmacists practicing in pharmacies with quotas for advanced clinical services and higher prescription volume were more likely to disagree that there was adequate staff support to provide safe and effective patient care. Pharmacist respondents had concerns regarding adequate time to perform job duties, time for breaks, and staff support. ${ }^{16}$ A follow-up study expanding the evaluation to additional provinces in Canada demonstrated a poor perception of working conditions among pharmacist respondents, which may contribute to occupational burnout. ${ }^{17}$

Based on the 2019 United States (U.S.) National Pharmacist Workforce Study, 6\% of pharmacists in the U.S. were working in an ambulatory care setting between 2014 and 2019. ${ }^{18}$ Pharmacist burnout was assessed using the Professional Fulfillment Index, that correlates with the MBI. ${ }^{19}$ They found that when comparing pharmacists working across all work settings, pharmacists working in ambulatory care have lower rates of work exhaustion, lower rates of interpersonal disengagement, and higher personal fulfillment. Ambulatory pharmacists scored the highest in the area of personal fulfillment when compared to any other patient care setting. ${ }^{18}$ Pharmacists' ability to practice to a fuller extent of their scope of practice in collaboration with other healthcare professionals may contribute to lower rates of burnout and higher rates of job satisfaction. The nature of primary care practice is conducive to long-term follow-up of patients compared to acute practice settings facilitating the formation of lasting patient-provider relationships. As previously identified by Maio and colleagues, pharmacists' job satisfaction varied by practice setting. ${ }^{20}$ Sixty-eight percent of pharmacists working in outpatient clinics, or similar practices in which long-term patient-provider relationships are founded, identified as being satisfied with their job. ${ }^{20}$ The long-term follow-up with patients in primary care practice may also contribute to the lower rates of burnout identified in this study.

The median score for the PA domain was relatively high at 5.0 (95\% Cl 4.69-5.22) out of a maximum score of 6 . When analyzed among the different categorical variables, it was determined that pharmacists who have worked in primary care for longer had a higher PA score. This indicated that those who are further into their career as a pharmacist in primary care have a greater sense of personal accomplishment. This could be explained by increased duration of time to build meaningful interprofessional relationships and trust while partaking in advanced practice activities such as quality assurance projects and practice-based research. A similar finding was observed in community pharmacists by Calgan and colleagues who demonstrated pharmacists working less than 10 years had low PA scores and those who had worked for greater than 30 years had the highest PA scores. ${ }^{6}$

The highest response rates were seen for the PA section with $90 \%$ completion. Completion rates were much lower for the depersonalization and emotional exhaustion sections. Although this tool is well known and widely used, the lack of response to particular questions could be an indication that the tool overall may not have resonated with respondents and thus may not be effective in addressing burnout in this particular population.

A major limitation of this study was the low response rate resulting in a small sample size. In addition, this was compounded by incomplete survey responses. Because there was no ability to mandate survey completion, valuable data was omitted precluding fulsome assessment of burnout rates in primary care pharmacists. Another limitation to assessing burnout at a national level was our ability to reach Canadian primary care pharmacists. The listserv used is populated by those who self-identify as primary care pharmacists, and as such, we cannot be sure that the survey has reached all those in our target population. As a way to mitigate non-primary care pharmacists from participating, it was clearly stated in the invitation to only complete the survey if they identified as a pharmacist practicing in team-based primary care. In addition, there is no official registry describing the number of current practicing primary care pharmacists to compare our sample to. The label "primary care pharmacist" was a limitation in itself because of the varying interpretations of the term across the country and even within regions. ${ }^{21}$ The survey results were also limited by the non-representativeness of the study population: $84 \%$ of respondents were female with a median age range of 45-54 years old and a median of $6-10$ years practicing in primary care. Lastly, the study was affected by response bias; those who were experiencing burnout may not have had the time or energy to complete the survey. Deploying the survey in December, typically a busy month for vacation, may have contributed to low response rates.

Future studies may consider alternate methods to identify team-based primary care pharmacists for improved representation and response rates. Additional areas to evaluate include details regarding the healthcare team and its potential effect on occupational burnout in pharmacists. As pharmacists may not be familiar with occupational burnout resources, it may be beneficial to specify burnout resources available at work to aid in identification. 


\section{CONCLUSION}

This is the first study to assess burnout among Canadian primary care pharmacists. Burnout rates could not be properly assessed due to the limited response rate. Personal accomplishment was higher in those who have been practicing in a primary care setting for longer. Future studies should consider alternate methods to evaluate burnout in this population. Additional studies evaluating occupational burnout in pharmacists across all practice settings Canada are required.

Availability of data and material: The data used and/or analysed in this study are available from the corresponding author upon reasonable request.

Ethics Approval: The study protocol and all components were reviewed and approved by the Behavioral Research Ethics Board of the Office of Research (Services) Ethics at the University of British Columbia (UBC) (study \# H19-03106).

Acknowledgement: We wish to thank Dr. Abdollah Safari from the Data Analytics, Statistics, and Informatics (DASI) unit at the University of British Columbia for statistical analysis consultation.

\section{REFERENCES}

1. Freudenberger HJ. Staff Burn-Out. Journal of Social Issues. 1974;30(1):159-165 DOI: 10.1111/j.15404560.1974.tb00706.x.

2. Bridgeman PJ, Bridgeman MB, Barone J. Burnout syndrome among healthcare professionals. American Journal of Health-System Pharmacy. 2018;75(3):147-152 DOI: 10.2146/ajhp170460.

3. World Health Organization. Burn-out an "occupational phenomenon": International Classification of Diseases. World Health Organization.

https://www.who.int/mental_health/evidence/burnout/en/. Published 2019. Accessed January 302020.

4. Maslach C, Jackson S, Leiter M. The Maslach Burnout Inventory Manual. In:1997:191-218.

5. El-Ibiary SY, Yam L, Lee KC. Assessment of Burnout and Associated Risk Factors Among Pharmacy Practice Faculty in the United States. American Journal of Pharmaceutical Education. 2017;81(4):75 DOI: 10.5688/ajpe81475.

6. Calgan Z, Aslan D, Yegenoglu S. Community pharmacists' burnout levels and related factors: an example from Turkey. International Journal of Clinical Pharmacy. 2011;33(1):92-100 DOI: 10.1007/s11096-010-9461-2.

7. Durham ME, Bush PW, Ball AM. Evidence of burnout in health-system pharmacists. American Journal of HealthSystem Pharmacy. 2018;75(23_Supplement_4):S93-S100 DOI: $10.2146 / a j h p 170818$.

8. Johnson RE, Ried LD, Robertson N. Self-Reported Burnout Among HMO Pharmacists. Journal of Pharmaceutical Marketing \& Management. 1987;2(2):107-127 DOI: 10.3109/J058v02n02_12.

9. Critical Care Medicine. 2019;47(1):34 DOI: 10.1097/01.ccm.0000550859.13262.4f.
10. Dolovich L, Pottie K, Kaczorowski J, et al. Integrating Family Medicine and Pharmacy to Advance Primary Care Therapeutics. Clinical Pharmacology \& Therapeutics. 2008;83(6):913-917 DOI: 10.1038/clpt.2008.29.

11. Government of Alberta. Primary health care. Government of Alberta. https://www.alberta.ca/primary-healthcare.aspx. Published 2014. Accessed February 13, 2020.

12. Maslach CJ, Susan. MBI - Human Services Survey for Medical Personnel - MBI-HSS (MP). Mind Garden Inc. www.mindgarden.com Published 2016. Accessed October 12, 2019.

13. Mind Garden. www.mindgarden.com. Accessed November 72019.

14. Mind Garden Inc. The Problem with Cut-Offs for the Maslach Burnout Inventory. Mind Garden Inc. https://www.mindgarden.com/documents/MBI-CutoffCaveat.pdf Published 2018. Accessed January 4, 2020.

15. Rotenstein LS, Torre M, Ramos MA, et al. Prevalence of Burnout Among Physicians: A Systematic Review. Jama. 2018;320(11):1131-1150 DOI: 10.1001/jama.2018.12777.

16. Tsao NW, Lynd LD, Gastonguay L, Li K, Nakagawa B, Marra CA. Factors associated with pharmacists' perceptions of their working conditions and safety and effectiveness of patient care. Canadian Pharmacists Journal / Revue des Pharmaciens du Canada. 2015;149(1):18-27 DOI: 10.1177/1715163515617777.

17. Tsao NW, Salmasi S, Li K, Nakagawa B, Lynd LD, Marra CA. Pharmacists' perceptions of their working conditions and the factors influencing this: Results from 5 Canadian provinces. Canadian Pharmacists Journal / Revue des Pharmaciens du Canada. 2020;153(3):161-169 DOI: 10.1177/1715163520915230.

18. Arya V, Bakken B, Doucette W, et al. National Pharmacist Workforce Study 2019. American Association of Colleges of Pharmacy https://www.aacp.org/sites/default/files/202003/2019_NPWS_Final_Report.pdf. Published 2020. Accessed September 282020.

19. Trockel $M$, Bohman B, Lesure E, et al. A Brief Instrument to Assess Both Burnout and Professional Fulfillment in Physicians: Reliability and Validity, Including Correlation with Self-Reported Medical Errors, in a Sample of Resident and Practicing Physicians. Acad Psychiatry. 2018;42(1):1124 DOI: 10.1007/s40596-017-0849-3.

20. Maio V, Goldfarb NI, Hartmann C. Pharmacists' Job Satisfaction: Variation by Practice Setting. Pharmacy and Therapeutics. 2004;29.

21. Tsuyuki RT, Beahm NP, Okada H, Al Hamarneh YN. Pharmacists as accessible primary health care providers: Review of the evidence. Can Pharm J (Ott). 2018;151(1):4-5 DOI: $10.1177 / 1715163517745517$. 
